\title{
Decisiones relevantes desde urgencias en la infección por la COVID-19: abordaje del paro cardíaco y respiratorio
}

Relevant decisions at emergency in COVID-19 infection: Approach to cardiac and respiratory arrest

\author{
Mateo Zuluaga-Gómez ${ }^{1 \text { × CvLAC-0RCID }}$, Deisy Tatiana Londoño-Londoño ${ }^{2}$, Santiago Upegui-Estrada ${ }^{3}$, \\ Jorge Iván Cohen-Cajiao" CvLAC
}

Fecha correspondencia: Recibido: abril 7 de 2020. Revisado: mayo 26 de 2020. Aceptado: julio 8 de 2020.

Forma de citar:

Zuluaga-Gómez M, LondoñoLondoño DT, Upegui-Estrada S, Cohen-Cajiao Jl. Decisiones relevantes desde urgencias en la infección por la COVID-19: abordaje del paro cardíaco y respiratorio. Rev CES Med. 2020; Especial COVID-19: 42-50.

Open access

(C) Derecho de autor

Licencia creative commons

Ética de publicaciones

Revisión por pares

Gestión por Open Journal System

DOl: http://dx.doi.org/10.21615/

cesmedicina.34.COVID-19.8

ISSN 0120-8705

e-ISSN 2215-9177

Comparte

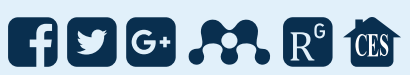

\section{Resumen}

La infección por coronavirus 2019 (SARS-CoV-2) ha conllevado a desenlaces fatales por compromiso cardiovascular o respiratorio, por lo que el personal de la salud debe estar atento y alerta a las recomendaciones sobre su abordaje y, al mismo tiempo, evitar su contaminación y contagio. Se hacen unas precisiones en cuanto al abordaje de la vía aérea y el paro circulatorio y cardíaco, que son diferentes al abordaje habitual de otros pacientes críticos. Se presenta una revisión de la literatura con información reportada hasta el momento sobre decisiones relevantes que puedan ser útiles en la intubación, protección personal, parámetros cardiovasculares y abordaje del paro cardiorrespiratorio.

Palabras clave: Infecciones; Coronavirus; Insuficiencia respiratoria; Paro cardíaco; Reanimación cardiopulmonar.

\begin{abstract}
Coronavirus 2019 infection (SARS-CoV-2) has led to fatal outcomes due to cardiovascular or respiratory involvement. So health care personnel must be attentive and alert to recommendations on how to deal with it while avoiding contamination and contagion. Some clarifications are made regarding the approach of the airway and circulatory and cardiac arrest, which are different from the usual approach of other critical patients. A review of the literature is presented with information reported so far on relevant decisions that may be useful in intubation, personal protection, cardiovascular parameters and approach to cardiorespiratory arrest.
\end{abstract}

Keywords: Infections; Coronavirus; Respiratory Insufficiency; Heart arrest, Cardiopulmonary Resuscitation

\section{Introducción}

La infección respiratoria por coronavirus 2019 se produce por el virus SARS-CoV-2 (por sus siglas en inglés, Severe Respiratory Acute Syndrome Coronavirus 2) (1,2). El virus pertenece a la subfamilia Orthocoronavirinae, un grupo de virus RNA al que pertenecen cuatro géneros: Alfa, Beta, Delta y 
Sobre los autores:

1. Residente Medicina de Urgencias. Estudiante Especialización Gerencia de IPS. Facultad de Medicina. Universidad CES, Medellín. Colombia.

2. Residente Medicina de Urgencias. Especialista Gerencia de IPS. Facultad de Medicina. Universidad CES, Medellín. Colombia.

3. Especialista en Medicina de Urgencias.

4. Coordinador programa de Especializacion en Medicina de Urgencias, Universidad CES. Especialista en Salud Pública. Docente Universidad CES. Coordinador CEMPAS. Universidad CES, Medellín, Colombia.

Línea de Investigación Medicina de Urgencias y Toxicología, Grupo de Investigación Especialidades Médicas y Quirúrgicas. Facultad de Medicina.
Gamma. Al género Betacoronavirus pertenece el SARS - CoV-2 (3-5). Se han descrito como órganos blanco el sistema cardiovascular y respiratorio, de allí la mayoría de las complicaciones sistémicas que pueden aparecer. Con este artículo se pretender mencionar generalidades de la pandemia, el comportamiento clínico, las complicaciones cardiovasculares y por ende las medidas a tener en cuenta desde el abordaje de la vía aérea y del paro cardiorrespiratorio en servicios de urgencias.

\section{Generalidades y evolución de la pandemia}

Los reportes de infección por SARS-CoV-2 se describen desde diciembre de 2019, cuando en Wuhan (China) se documentan 27 casos de neumonía sin una etiología clara que tras aislamiento genómico sería lo que se conoce actualmente como la COVID-19 (6). Posteriormente, con aparición de miles de casos en todo el mundo el 11 de febrero de 2020 la Organización Mundial de la Salud establece la denominación internacional de acuerdo con la taxonomía viral, como SARS-COVID-19 (7) y es declarado como pandemia el 11 de marzo (8). En Colombia, a partir del 6 de marzo de 2020 se confirma el primer caso y, a la fecha del reporte y correcciones de este manuscrito (18 de junio), con información del Instituto Nacional de Salud hay 57046 casos y 1864 muertes confirmadas (8).

\section{Presentación, transmisión y manifestaciones clínicas}

El periodo de incubación de la infección oscila entre 1 a 14 días, dependiendo de la inmunidad de la persona infectada $(9,10)$. El origen de transmisión descrita es por gotas (partículas de 0,5 micras) que llegan hasta una distancia de 1,8 metros, e incluso aerosoles (10 metros) pues el virus puede quedar libre en el aire. El inicio de los síntomas es entre cinco y nueve días posteriores al contagio (cuadro 1) (11).

Cuadro 1. Manifestaciones clínicas de COVID-19

\begin{tabular}{|c|c|}
\hline Manifestación & $\%$ \\
\hline Fiebre & $83-98$ \\
\hline Tos seca & $59-82$ \\
\hline Disnea* & $31-55$ \\
\hline Síndrome de dificultad respiratoria aguda " (SDRA) & 17 \\
\hline Fatiga & $44-69$ \\
\hline Mialgias & $11-44$ \\
\hline Tos con expectoración & $26-28$ \\
\hline Confusión & 9 \\
\hline Cefalea & $6-8$ \\
\hline Rinorrea & 4 \\
\hline Dolor torácico & $2-5$ \\
\hline Síntomas gastrointestinales ${ }^{* * *}$ & $1-10$ \\
\hline Ataque cerebrovascular agudo ${ }^{+}$ & 5,7 \\
\hline \multicolumn{2}{|c|}{$\begin{array}{l}\text { * Suele aparecer entre el día } 5 \text { y } 8 \text { posterior al contagio. } \\
{ }^{* *} \text { El debut con SDRA suele occurrir en pacientes con factores de riesgo o comorbilidades (enfermeda } \\
\text { coronaria, hipertensión arterial, cáncer, insuficiencia cardíaca). } \\
{ }^{* * *} \text { Usualmente diarrea y vómito incoercibles. } \\
\text { + No es clara la etiología y asociación, pero se ha descrito un riesgo aumentado de ataque cerebrovas } \\
\text { cular debido a precipitación de eventos cardioembólicos asociados. }\end{array}$} \\
\hline
\end{tabular}




\section{COVID-19 y sistema cardiovascular}

Poco se conoce de las manifestaciones cardiovasculares de la infección por SARS-CoV 2 que se presentan en el contexto de aumento en la respuesta inflamatoria sistémica que ejerce el virus en sistema inmune (12-14). De acuerdo con estudios en población oriental, los mayores de 65 años tendrán mayor probabilidad de presentar complicaciones, principalmente aquellos con hipertensión arterial, diabetes mellitus tipo 2 o enfermedad coronaria preexistente $(9,14)$.

La letalidad en pacientes con distintas comorbilidades es superior a aquellos que no pesentan enfermedad cardiovascular (10,5\%), cáncer (5,6\%), hipertensión arterial (6\%), enfermedad pulmonar obstructiva crónica (6,3\%), diabetes mellitus tipo 2 (7,3 \%). Estos pacientes presentan mayor riesgo de lesión miocárdica, isquemia, inflamación, choque cardiogénico, hipoxemia grave, hipertensión pulmonar, insuficiencia cardíaca y paro cardiopulmonar $(15,16)$. Las hipótesis asociadas incluyen la posible unión al receptor de la enzima convertidora de angiotensina (ACE-2), aumento de citocinas mediadas por linfocitos $T$ tipo 1 y 2, hipoxemia por disfunción pulmonar y lesión miocárdica $(17,18)$.

Las indicaciones de realizar ventilación mecánica a un paciente con infección por la COVID-19 son: relación $\mathrm{PaO} 2 /$ $\mathrm{FiO} 2<150$ o $<200$ en pacientes mayores de 60 años; frecuencia respiratoria mayor a 30 por minuto; falla ventilatoria hipercápnica; escala de Glasgow < 8; no mejoría de la saturación $(<90 \%)$ con cánula de alto flujo.
En una cohorte prospectiva de 416 pacientes con diagnóstico confirmado de la COVID-19 entre enero y febrero de 2020, en un hospital de China, se encontró que aquellos con lesión miocárdica tenían mayor edad (mediana 74 años RIQ 34 - 95 años vs 60 años RIQ 21 - 90 años), mayor número de comorbilidades (enfermedad coronaria, ataque cerebrovascular, falla cardíaca crónica, cáncer). La tasa de mortalidad fue mayor en pacientes con lesión miocárdica y elevación de la troponina comparado con quienes no presentaban lesión miocárdica $(51,2 \%$ vs 4,5$)$, el riesgo ajustado para muerte en pacientes que presentaban lesión miocárdica fue cuatro veces mayor (HR 4,3; IC95 \% 1,9-9,5). Se describen limitaciones en la validez del estudio por pérdida de información de covariables (19).

Otro metaanálisis reportado frente a la asociación de niveles de troponina con gravedad clínica en pacientes con la COVID-19, incluye 341 pacientes, de ellos el $36 \%$ presentaban manifestaciones graves, y tenían niveles de troponina más altos comparado con quienes tenían manifestaciones leves (diferencia estandarizada de medias de 25,6 ng/L, IC 95 \% 6,8 - 44,5ng/L); también con limitaciones en la validez interna de los estudios incluidos. La medición de troponina no precede a la progresión de la enfermedad y la elevación puede deberse a otros mecanismos $(20,21)$.

\section{Abordaje de la vía aérea en situaciones de falla ventilatoria}

Debido a la exposición a secreciones y el riesgo de contagio el abordaje de la vía aérea debe realizarse con todas las premisas de bioseguridad y cuidado. Las indicaciones de realizar ventilación mecánica a un paciente con infección por la COVID-19 son (22-24):

- Relación presión arterial de oxígeno / fracción inspirada de oxígeno $\left(\mathrm{PaO}_{2} / \mathrm{FiO}_{2}\right)$ $<150$ o $<200$ en pacientes mayores de 60 años.

- Frecuencia respiratoria mayor a 30 por minuto.

- Falla ventilatoria hipercápnica (presión arterial de $\mathrm{CO}_{2} ; \mathrm{PaCO}_{2}>50 \mathrm{~mm} \mathrm{Hg}$ ).

- Escala de Glasgow $<8$.

- No mejoría de la saturación (<90\%) con cánula nasal de alto flujo (60 litros por minuto por al menos dos horas) o de acuerdo con el dispositivo utilizado. 
Cuadro 2. Procedimiento generadores de aerosoles

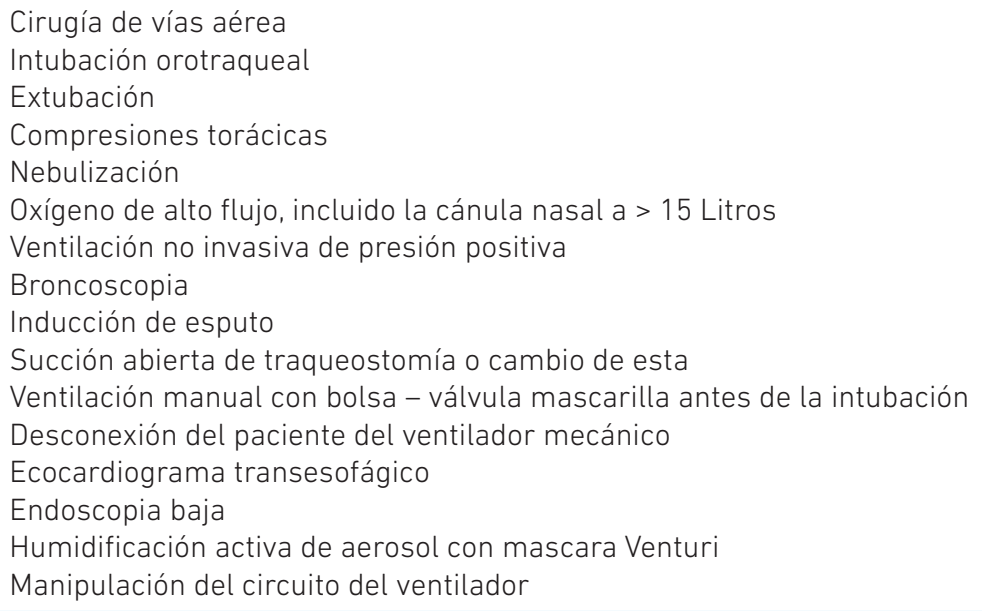

Se sugiere intubar a partir del uso de video laringoscopio como estrategia difícil en pacientes con la COVID-19. Por su poca disponibilidad, se recomienda la laringoscopia directa con doble guante y retirar inmediatamente el guante externo luego de la intubación.
La técnica de secuencia rápida de intubación se implementará así (25):

- Preoxigenación al menos cinco minutos, con una fracción inspirada de oxígeno $\left(\mathrm{FiO}_{2}\right)$ del $100 \%$, evitando el uso de ventilación por presión positiva.

- La World Federation of Societies of Anaesthesiologists (WFSA, por sus siglas en inglés) sugiere intubar a partir del uso de video laringoscopio como estrategia de intubación por excelencia, teniendo en cuenta la consideración de vía aérea difícil en pacientes con la COVID-19. Por la poca disponibilidad, se recomienda la laringoscopia directa con doble guante y retirar inmediatamente el guante externo luego de la intubación (26).

- Conexión de dispositivo BVM (bolsa - válvula - máscara) a un filtro que logre separar el tubo endotraqueal del paciente. Deben tenerse todos los circuitos listos y preparados, y en caso de contar con ventilador debe estar en modo espera con parámetros de ventilación protectora $(23,25)$.

- No se recomienda el uso de máscara laríngea o fibrobroncoscopio (23,26).

- Se recomienda el empleo de dispositivos para verificación secundaria del acceso de la vía aérea como el capnógrafo o el dispositivo colorimétrico de acuerdo a la disponibilidad. En el servicio de urgencias, debido a su baja disponibilidad, se recomienda continuar con métodos como verificación primaria (paso por cuerdas vocales) y verificación secundaria (26).

- Se recomienda el uso de dispositivos como la caja de Taiwan (o aerosol Box) que limita la contaminación con las partículas en aerosol durante el proceso de intubación $(27,28)$. Debe considerarse que puede aumentar los tiempos de intubación y de hipoxia y generar daños en el equipo de protección convencional. Se requiere mayor investigación para garantizar su uso seguro en la práctica clínica (29). 


\section{Reanimación cardiopulmonar y otras medidas durante la resu- citación}

El probable ritmo cardíaco que se esperaría encontrar en un paciente con infección por la COVID-19 será un ritmo no desfibrilable, actividad eléctrica sin pulso (AESP) o asistolia, debido a la posible hipoxia; sin descartar, ante el compromiso miocárdico o enfermedad coexistente, la presentación de un ritmo desfibrilable $(30,31,33)$. Las prioridades en estos pacientes irán encaminadas a $(25,26,31)$ :

- Todo el personal debe continuar y seguir las directrices nacionales del uso de equipo de protección personal previo al contacto con el paciente. Se deberá utilizar mascarilla N95, mascarilla desechable sobre la mascarilla de alta eficiencia, batas desechables, delantal plástico, visor o protección ocular.

- Reducir al mínimo el número de personas para la atención del paciente (figura 1): un médico (el de mayor formación académica) será el líder del equipo e idealmente abordará la vía aérea del paciente. Deben evitarse fallas en el proceso de ventilación e intubación al igual que el número de intentos, por lo cual será el más entrenado de acuerdo al escenario (en urgencias se propone que sea el

Reducir al mínimo el número de personas para la atención del paciente. médico especialista en urgencias); un profesional de enfermería (para la administración de medicamentos), dos auxiliares de enfermería (de acuerdo a la disponibilidad del recurso de talento humano durante el turno), uno de ellos será circulante y el otro apoyará las compresiones torácicas.

- Reconocer el paro cardíaco y activar tempranamente el código azul.

- Tener prioridad en el inicio de las compresiones torácicas (técnica Hands Only CPR, por sus siglas en inglés) por el primer participante que ya esté con todo el equipo de protección personal.

- Evitar la ventilación boca a boca o con dispositivo bolsa - válvula -mascarilla, por riesgo de exposición a fluidos corporales.

- Desechar y limpiar todo el equipo utilizado de acuerdo con las directrices locales. Utilizando técnica de doble guante y en uno de ellos disponer el material para desinfección o desecho.

- Lavado de manos al final de la reanimación para evitar propagación. 
La atención de pacientes en una pandemia debe ser guiada a partir de consideraciones éticas y de salud pública, al igual que la priorización del uso de los elementos de protección personal y la disponibilidad de los recursos. En caso de complicaciones o mala evolución se podrá plantear una limitación al esfuerzo terapéutico, evitando la futilidad.
PARO RESPIRATORIO Y CIRCULATORIO

En persona con sospecha o confirmación de SARS CoV- 2

I

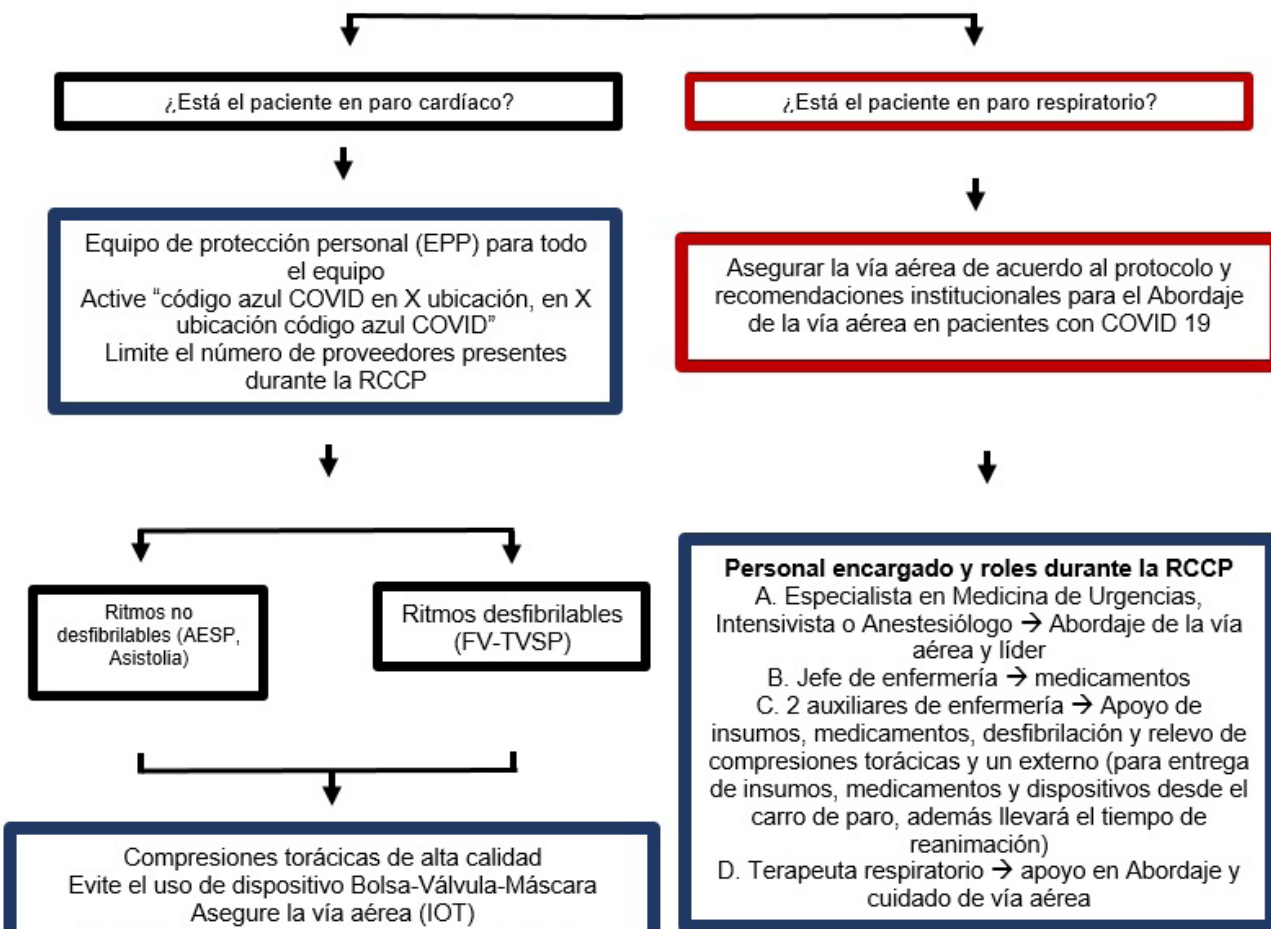

Administre adrenalina $1 \mathrm{mg}$ IV cada 3 - 5 minutos

Verifique ritmo cada 2 minutos, en caso de cambio de ritmo verifique pulso.

En caso de ritmo desfibrilable administre una descarga según el equipo (Monofásico 360J / Bifásico 220J)

Figura 1. Paro respiratorio y circulatorio

Figurada diseñado por los autores del manuscrito. Abril de 2020.

AESP: Actividad Eléctrica Sin Pulso, FV: Fibrilación Ventricular, TV: Taquicardia Ventricular Sin Pulso, IOT: Intubación Orotraqueal

\section{Decisiones éticas: reanimación en condición de pandemia}

La atención de pacientes en una pandemia debe ser guiada a partir de consideraciones éticas y de salud pública, al igual que la priorización del uso de los elementos de protección personal y la disponibilidad de los recursos $(34,35)$. En caso de complicaciones o mala evolución se podrá plantear una limitación al esfuerzo terapéutico, evitando la futilidad $(36,37)$.

\section{Declaración conflictos de interés}

Los autores declaran autoría completa de este manuscrito y no presentan algún conflicto de interés.

\section{Bibliografía}

1. Millan-Oñate J, Rodríguez-Morales AJ, Camacho-Moreno G, Mendoza-Ramírez $\mathrm{H}$, Rodríguez-Sabogal IA, Álvarez-Moreno C. A new emerging zoonotic virus of concern: the 2019 novel Coronavirus (COVID-19). Infectio 2020;24 (3):1 - 6.

2. Chen N, Zhou M, Dong X, Qu J, Gong F, Han Y, et al. Epidemiological and clinical characteristics of 99 cases of 2019 novel coronavirus pneumonia in Wuhan, China: a descriptive study. Lancet 2020; 395: 507 - 13. 
3. Huang C, Wang Y, Li X, Ren L, Zhao J, Hu Y, et al. Clinical features of patients infected with 2019 novel coronavirus in Wuhan, China. Lancet 2020;395:497-506.

4. Li Q, Guan X, Wu P, Wang X, Zhou L, Tong Y, et al. Early transmission dynamics in Wuhan, China, of novel coronavirus-infected pneumonia. N Engl J Med 2020: 386 (13): 1199.

5. Munster VJ, Koopmans M, van Doremalen N, van Riel D, de Wit E. A Novel Coronavirus Emerging in China - Key Questions for Impact Assessment. N Engl J Med. 2020;382:692-94.

6. World Health Organization. Novel Coronavirus (2019-nCoV) - Situation report - 7 - 27 January 2020. [Sitio en Internet] Hallado en URL: https://www.who.int/docs/ default-source/coronaviruse/situationreports/20200127-sitrep-7-2019-ncov. pdf?sfvrsn=98ef79f5_2020. 2020. Acceso en marzo 25 de 2020.

7. World Health Organization. Pneumonia of unknown cause - China. [Sitio en Internet] Hallado en URL: https://www.who.int/csr/don/05-january-2020-pneumonia-of-unkown-causechinal. Acceso en Marzo 25 de 2020.

8. Instituto Nacional de Salud. Coronavirus (COVID-19) en Colombia. [Sitio en Internet] Hallado en URL: https://www.ins.gov.co/Noticias/Paginas/Coronavirus. aspx. Acceso en junio 17 de 2020.

9. Yang X, Yu Y, Xu J, Shu H, Xia Ja, Liu H, et al. Clinical course and outcomes of critically ill patients with SARS-CoV-2 pneumonia in Wuhan, China: a single-centered, retrospective, observational study. Lancet Respir Med 2020; 8 (5): 475 -481.

10. Rothe C, Schunk M, Sothmann P, Bretzel G, Froeschl G, Wallrauch C, et al. Transmission of 2019-nCoV infection from an asymptomatic contact in Germany. N Engl J Med 2020; 382 (10): 970 - 971.

11. Liang T. Handbook of CODIV - 19. Prevention and treatment. [Sitio en Internet] Hallado en URL: https://cm-us-standard.s3.amazonaws.com/documents/Zhejiang-University-Handbook-of-COVID-19-Prevention-and-Treatment.pdf. Acceso en Marzo 24 de 2020.

12. Gallego A, Remolina S, Méndez I. A propósito del Coronavirus - COVID 19 ¿Que pasa con el sistema cardiovascular? Puesta al día en Cardiología - Boletin 139. [Sitio en Internet] Hallado en URL: : http://scc.org.co/boletin-no-139/ Acceso en Marzo 24 de 2020

13. Roujian Lu, Xiang Zhao, Juan Li, Peihua Niu, Bo Yang, Honglong, et al. Genomic characterisation and epidemiology of 2019 novel coronavirus: implications for virus origins and receptor binding. Lancet 2020; 395 (10224): 565-574.

14. Zheng Y, Ma Y, Zhang J. COVID-19 and the cardiovascular system. Nat Rev Cardiol 2020; 17 (5): $259-260$.

15. Multicenter collaboration group of Department of Science and Technology of Guangdong Province and Health Commission of Guangdong Province for chloroquine in the treatment of novel coronavirus pneumonia. [Expert consensus 
on chloroquine phosphate for the treatment of novel coronavirus pneumonia]. Zhonghua Jie He Hu Xi Za Zhi; 2020:43(3):185-188.

16. Wang M, Cao R., Zhang L, Yang X, Liu J, Xu M, et al. Remdesivir and chloroquine effectively inhibit the recently emerged novel coronavirus (2019-nCoV) in vitro. Cell Res 2020; 30, 269-271.

17. Huang C, Wang Y, Li X, Ren L, Zhao J, Hu Y,et al. Clinical features of patients infected with 2019 novel coronavirus in Wuhan, China. Lancet 2020; 395, 497-506.

18. Diagnostic and Interventional Cardiology. ACC COVID-19 Clinical Guidance For the Cardiovascular Care Team. [Sitio en Internet] Hallado en URL: https://www.dicardiology.com/article/acc-covid-19-clinical-guidance-cardiovascular-care-team. Acceso en Marzo 24 de 2020.

19. Shi S, Qin M, Shen B, Cai Y, Liu T, Yang F, et al. Association of cardiac injury with mortality in hospitalized patients with COVID-19 in Wuhan, China. JAMA Cardiol. 2020 Mar 25. doi: 10.1001/jamacardio.2020.0950

20. Giuseppe Lippi, Carl J. Lavie, Fabian Sanchis-Gomar. Cardiac troponin I in patients with coronavirus disease 2019 (COVID-19): Evidence from a meta-analysis. Prog Cardiovasc Dis. 2020. doi: 10.1016/j.pcad.2020.03.001.

21. Diagnostic and Interventional Cardiology. ESC Council on Hypertension Says ACE-I and ARBs Do Not Increase COVID-19 Mortality. [Sitio en Internet] Hallado en URL: https://www.dicardiology.com/content/esc-council-hypertension-says-ace-iand-arbs-do-not-increase-covid-19-mortality. Acceso en Marzo 24 de 2020.

22. Greenland JR, Michelow MD, Wang L, London MJ. COVID-19 Infection: implications for perioperative and critical care physicians. Anesthesiology 2020; 132 (6): $1346-1361$.

23. Xiangdon C, Liu Y, Gong Y, Guo Z, Zuo M. Perioperative management of patients infect with the novel coronavirus. Anesthesiology 2020; 132(6): 1307 - 1316.

24. Tran K, Tran K, Cimon K, Severn M, Pessoa-Silva CL, Conly J. Aerosol generating procedures and risk of transmission of acute respiratory infections to healthcare workers: a systematic review. PLoS One. 2012;7(4):e35797

25. Cheung JC-H, Ho LT, Cheng JV, Cham EYK, Lam KN. Staff safety during emergency airway management for COVID-19 in Hong Kong. Lancet Respir Med 202; 8 (4): e19.

26. Tang G, Ming A. Perioperative management of suspected/confirmed cases of COVID-19. [Sitio en Internet] Hallado en URL: https://www.wfsahq.org/components/com virtual library/media/1c4ec5c64b9aaacf7c47f76a61fb6edc-atow-422-01.pdf. Acceso en Junio 17 de 2020.

27. Resuscitation Council UK. Resuscitation Council UK Statement on COVID-19 in relation to CPR and resuscitation in first aid and community settings. [Sitio en Internet] Hallado en URL: https://www.resus.org.uk/media/statements/resuscitation-council-uk-statements-on-covid-19-coronavirus-cpr-and-resuscitation/ covid-community/. Acceso en Marzo 24 de 2020. 
28. Public Health Wales statement on Novel Coronavirus (COVID-19) outbreak: [Sitio en Internet] Hallado en URL: https://phw.nhs.wales/news/public-health-wales-statement-on-novel-coronavirus-outbreak. Acceso en Marzo 24 de 2020.

29. Lai HY, Chang D. Aerosol box protects healthcare providers during endotracheal intubation. [Sitio en Internet] Hallado en URL: https://sites.google.com/view/aerosolbox/home. Acceso en Junio 16 de 2020

30. Malik J, Jenner C, Ward P. Maximising Application of the aerosol box in protecting healthcare workers during the COVID-19 Pandemic. Anaesthesia 2020, 75 (7): $974-975$.

31. Begley J, Lavery K, Nickson C. The aerosol box for intubation in COVID-19 patients: an in situ simulation crossover study. Anaesthesia 2020. Doi: 10.1111/ anae.15115. doi: 10.1111/anae.15115.

32. Coronavirus (Covid-19) updates for Northern Ireland. [Sitio en Internet] Hallado en URL: https://www.health-ni.gov.uk/coronavirus\. Acceso en Marzo 27 de 2020.

33. COVID-19: guidance for health professionals [Public Health England]. [Sitio en Internet] Hallado en URL: https://www.gov.uk/government/collections/wuhan-novel-coronavirus. Acceso en Marzo 27 de 2020.

34. Coronavirus (Covid-19) [Health Protection Scotland]. [Sitio en Internet] Hallado en URL: https://www.hps.scot.nhs.uk/a-to-z-of-topics/covid-19/. Acceso en Marzo 27 de 2020.

35. Edelson D, Sasson C, Chan P, Atkins D, Aziz K, Becker L, et al. Interim Guidance for Basic and Advanced Life Support in Adults, Children, and Neonates With Suspected or Confirmed COVID-19. [Sitio en Internet]. Hallado en URL: https://www. ahajournals.org/doi/pdf/10.1161/CIRCULATIONAHA.120.047463. Acceso en Junio 15 de 2020.

36. Sociedad Española de Geriatría y Gerontología. Recomendaciones generales relacionadas con las decisiones éticas difíciles y la adecuación de la intensidad asistencial. [Sitio en Internet] Hallado en URL: https://www.segq.es/media/descargas/CONSENSO-RECOMENDACIONES-UCI-COVID-19.pdf. Acceso en Marzo 22 de 2020.

37. White, DB, Luce J, Lo B.. Who should receive life support during a public health emergency? Using ethical principles to improve allocation. Ann Intern Med 2009; 150: $132-138$

38. Saxena, A. Horny P, Amuasi J, Aagaard N, Kohler J, Gooshki S, et al. Ethics preparedness: Facilitating ethics review during outbreaks. BMC Med Ethic. 2019; 20, $1-10$.

39. Grasselli G, Pesenti A, Cecconi M. Critical Care Utilization for the COVID-19 Outbreak in Lombardy, Italy. Early experience and forecast during an emergency response. JAMA. 2020; doi: 10.1001/jama.2020.4031. 